\title{
Identification of Mitochondrial-related Prognostic Biomarkers Associated with Metabolic Processes of Hepatocellular Carcinoma
}

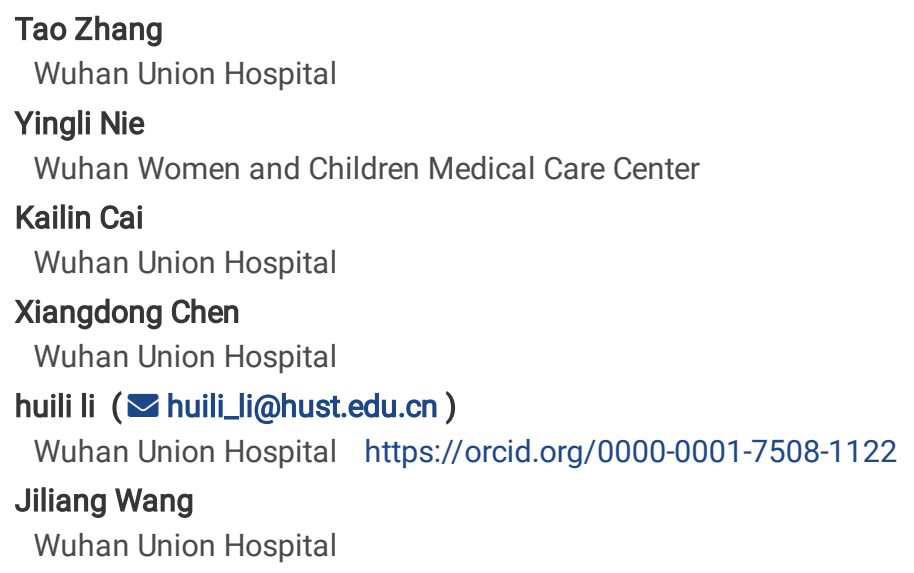

Keywords: hepatocellular carcinoma, mitochondria, prognosis, biomarker, metabolism

Posted Date: May 29th, 2020

DOI: https://doi.org/10.21203/rs.3.rs-30885/v1

License: (1) This work is licensed under a Creative Commons Attribution 4.0 International License. Read Full License 


\section{Abstract Background}

Hepatocellular carcinoma (HCC) is one of the leading causes of tumor-associated deaths worldwide. Despite the great progress in early diagnosis and multidisciplinary tumor management, the long-term prognosis of HCC remains unfavorable. Currently, metabolic reprogramming is widely found during tumor development to satisfy the growth and proliferation of cancer cells. And several metabolic targets have been identified and used as cancer biomarkers. Liver and mitochondrion are the two hubs of metabolism in humans in the organic and cellular levels, respectively. Thus, identifying prognostic biomarkers in mitochondrial-related genes (Mito-RGs) that reflect the metabolic alteration of HCC could lead to better interventions for HCC patients.

\section{Methods}

mRNA expression profiles and clinicopathological data of hepatocellular carcinoma (HCC) were obtained from The Cancer Genome Atlas (TCGA) database and Mito-RGs were obtained from mSigDB database. Weighted gene coexpression network analysis (WGCNA) was used to identify the interesting gene module that associated with HCC prognosis. Based on a univariate and the least absolute shrinkage and selection operation (LASSO) Cox regression analyses, a Mito-RGs based classifier was established and used for predicting the overall survival (OS) in HCC patients. Receiver operating characteristic (ROC) curve and multivariate Cox regression analysis were used to evaluate the prognosis performance of our classifier. Gene Set Enrichment Analysis (GSEA), Gene Set Variation Analysis (GSVA) and Consensus ClusterPlus algorithms were used to identify metabolic pathways that were significantly different between the high and low-risk groups based on the risk score calculated by the classifier.

\section{Results}

We constructed a classifier containing 10 Mito-RGs (ACOT7, ADPRHL2, ATAD3A, BSG, FAM72A, PDK3, PDSS1, RAD51C, TOMM34, and TRMU) for predicting the prognosis of HCC. And the classifier is an independent prognostic signature in predicting HCC patients' survival. Besides, the metabolic processes about fatty acid, amino acid, and bile acid were significantly downregulated in high-risk group, which may play notable roles in the development and unfavorable prognosis of HCC.

\section{Conclusion}

The Mito-RGs based classifier could act as an independent biomarker for survival prediction of HCC patients. Moreover, our research may provide a novel method for HCC metabolic therapy based on the modulation of mitochondrial function.

\section{Background}

Hepatocellular carcinoma (HCC) is one of the most common malignant cancers and is currently the fifth and seventh leading cause of cancerrelated deaths worldwide in females and males, respectively [1]. Despite the great progress that has been made in early diagnosis and multidisciplinary tumor management, the long-term prognosis remains unfavorable. Therefore, novel and effective prognostic models are needed to improve clinical management by identifying patients with high risks of unfavorable prognosis. Conventional models using clinical TNM stage, vascular invasion, and other parameters to help to predict the prognosis of HCC patients. However, considering the high heterogeneity of HCC in morphological and biological, the prediction efficacy of these models is still unsatisfying.

Mitochondria are the hub of cellular metabolism that regulates substance and energy metabolism that are essential for cell growth, proliferation, differentiation, and death [2]. Therefore, mitochondria are deeply involved in various cancer biological processes, including cancer initiation, growth, invasion and metastasis, recurrence, and resistance to drugs [3]. The mutation and epigenetic modulation of mitochondrial DNA, the reprogramming of energy metabolism, and the alteration of mitochondrial channels were found to play vital roles in cancer biology [3]. Emerging studies have demonstrated that mitochondrial metabolism is potentially a promising target for cancer therapy [4], since various mitochondrial metabolic processes are altered in tumors [5]. Thus, it is of vital importance to take mitochondrial-related biomarkers into account to establish a novel predictive tool.

The liver is the key regulator that manages whole-body metabolism and maintains metabolic homeostasis. Recent studies demonstrated that substantial metabolic alterations are present in liver diseases and contribute to HCC development. Besides, the metabolic rearrangements in HCC, making it unique and challenging malignancy [6]. Thus, the application of mitochondrial-related genes (Mito-RGs)-based prognostic 
biomarkers in HCC is a potential. Furthermore, based on this, we can explore the underlying metabolic alterations and identify potential therapeutic drugs, so as to bring new insights into the improvement of the prognosis of HCC patients.

In the present study, we constructed a classifier containing 10 Mito-RGs for HCC overall survival by utilizing the least absolute shrinkage and selection operation (LASSO) Cox regression. Based on the risk score calculated by the classifier, the samples were divided into low and highrisk groups. We further investigated the metabolism alterations and metabolic subgrouping of HCC samples by Gene Set Variation Analysis (GSVA) and Consensus ClusterPlus. Our results demonstrate that the Mito-RGs-based classifier could be used as a reliable prognostic predictor for HCC survival, and the suppression of metabolic processes about fatty acid, amino acid, and bile acid may play vital roles in the development and poor prognosis of HCC. Moreover, our research may provide a novel method for HCC metabolic therapy based on the modulation of mitochondrial function.

\section{Materials And Methods}

\subsection{Data Source and Pre-processing}

An RNA-seq dataset and the corresponding clinical parameters of HCC tissues $(n=374)$ and normal liver tissues ( $n=50)$ were downloaded from UCSC-Xena (https://xenabrowser.net/datapages/) based on TCGA database. The HCC patients with complete survival data and RNA-seq data were included in the subsequent analysis. HCC data were annotated by Homo_sapiens.GRCh38.84.chr.gtf (ftp.ensembl.org) file in this study.

Mito-RGs in the present study were defined as the mitochondrial-located protein-coding genes. The genesets of these genes were obtained from CC: GO cellular component of mSigDB (http://software.broadinstitute.org/gsea/msigdb) in Feb. 2020 . A total of 23 genesets and 1568 unique genes were screened finally (Additional files 1:Supplementary table 1).

\subsection{Weighted Gene Coexpression Network Construction and Interesting Module Detection}

Weighted gene coexpression network analysis (WGCNA) for all Mito-RGs in the HCC dataset was performed according to the protocols of WGCNA [7], described previously [8]. Briefly, every pairwise gene-gene relationship was calculated in the first step. Then, a 'soft' power adjacency function was utilized to construct the topological overlap matrix (TOM) and adjacency matrix. Clusters of genes with high topological overlap were identified as "Gene modules", utilizing a dissimilarity measure (1-TOM).

The correlations between modules and clinical characteristics were calculated by Pearson's correlation tests to identify modules with significantly clinical meanings. The modules that exhibited high correlations with HCC clinical characteristics were selected as interesting modules to be further studied.

\subsection{Identification of Prognostic Mito-RGs}

A univariate Cox regression was performed for all Mito-RGs in interesting modules and the genes with $\mathrm{P}<0.05$ were identified as prognostic Mito-RGs.

\subsection{Establishment of Prognostic Classifier}

The LASSO Cox regression analysis was applied for all prognostic Mito-RGs to select the most useful prognostic biomarkers and to construct the survival-predicting classifier. The prognosis risk scores were calculated based on a formula as follows:

RiskSocre $=\Sigma$ (Genes cox coefficient $\times$ Genes expression levels $)$

We then utilized the cut-off of the median risk score to divided the HCC patients into low and high-risk groups. The predictive capacity of the model for the training and validation cohort, which randomly split at a 1:1 ratio, as well as the total cohort was evaluated by the Kaplan-Meier log-rank test. In addition, the application value of the model was tested by receiver operating characteristic (ROC) curve analysis, and by univariate and multivariate Cox regression analysis.

\subsection{Pathway Enrichment Analysis}

In order to investigate significantly altered metabolic pathways between high and low risk groups, we performed Gene Set Enrichment Analysis (GSEA) analysis. The clusterProfiler R package [9] was used to perform GSEA analysis based on Kyoto Encyclopedia of Genes and Genomes (KEGG) database between high and low-risk groups. KEGG analysis was using the cut-off value of $\mathrm{P}<0.05$.

\subsection{Gene Set Variation Analysis (GSVA)}


To further reveal pathway enrichment between the two groups, we conducted GSVA [10] to evaluate pathway activity conditions. We selected C2.CP:KEGG.V7.0.symbols.gmt file, consisting of 186 KEGG genesets, as the reference gene set file. Then, the GSVA package was implemented for these 186 gene sets to further obtain the GSVA scores of each geneset for each sample, which devotes the degree of absolute enrichment of a gene set in each sample. After that, we used limma, and pheatmap packages to display distinct pathways between high and low-risk groups.

\subsection{Metabolic subgrouping}

To cluster HCC samples into different metabolic subgroups, we used the Consensus ClusterPlus R package [11] to select the optimal cluster number (parameters: reps $=1000$, pltem $=0.8$, pFeature $=1$ ). The survival and GSVA analyses were then performed in different subgroups.

\subsection{Statistical Analysis}

All statistical analyses were conducted by R version 3.6.1 and SPSS version 22.0 software. The correlation between risk score and clinicopathological characteristics was analyzed by the chi-square test. The statistical significance for normally distributed variables of the two groups was estimated by the two-tailed unpaired t-test. $\mathrm{P}<0.05$ were considered statistically significant.

\section{Results}

\subsection{Coexpression Network Construction and Interesting Module Detection}

WGCNA was conducted on 1519 Mito-RGs in the 374 HCC samples. When the soft-threshold power $\beta$ was set to 8 , a scale-free network distribution was formed of the connectivity between the genes in the gene network (Fig. 1a). Then, 7 coexpressed modules were identified (Fig. 1b). The correlations between modules and clinical features, such as gender, age, child-pugh grade, BMI, histologic grade, pathologic T, pathologic $\mathrm{N}$, pathologic $\mathrm{M}$, tumor stage, vital status, and days to death were calculated. The red module was highly correlated with histologic grade $\left(r=0.24, P=3 \cdot 10^{-6}\right)$, pathologic $T\left(r=0.24, P=5 \cdot 10^{-6}\right)$, tumor stage $\left(r=0.23, P=1 \cdot 10^{-5}\right)$, vital status $\left(r=0.21, P=4 \cdot 10^{-5}\right)$, and days to death $\left(r=-0.21, P=P=5 \cdot 10^{-5}\right)$ (Fig. 1c). Thus, the red module was selected as an interesting module to be studied in subsequent analyses.

\subsection{Identification of Prognostic Mito-RGs}

Univariate Cox regression for all Mito-RGs in red $(n=63)$ was conducted (Additional files 2: Supplementary table 2) and identified 49 genes with $\mathrm{P}<0.05$ as prognostic Mito-RGs (Additional files 2: Supplementary Fig. 1).

\subsection{Establishment of Prognostic Classifier}

LASSO Cox regression analysis was conducted to choose the most useful prognostic biomarkers for constructing the prognostic-predicting classifier base on the training cohort (Fig. 2a). A total of 10 Mito-RGs (ACOT7, ADPRHL2, ATAD3A, BSG, FAM72A, PDK3, PDSS1, RAD51C, TOMM34, and TRMU) were identified as the most useful prognostic biomarkers (Table 1).

Table 1

The mitochondrial-related genes in the prognostic classifier associated with LIHC in the TCGA dataset

\begin{tabular}{|lllll|}
\hline \multirow{2}{*}{ Symbol } & \multicolumn{2}{l}{ Univariate Cox regression analysis } & \multicolumn{2}{l}{ LASSO } \\
\cline { 2 - 4 } & HR & $95 \% \mathrm{Cl}$ & P-value & Coefficient \\
\hline ACOT7 & 34.213 & $6.863-170.547$ & 0.000 & 0.562 \\
\hline ADPRHL2 & 293.836 & $41.093-2101.042$ & 0.000 & 0.767 \\
\hline ATAD3A & 51.854 & $9.832-273.46$ & 0.000 & 0.211 \\
\hline BSG & 60.209 & $8.929-405.992$ & 0.000 & 1.226 \\
\hline FAM72A & 2.484 & $1.521-4.056$ & 0.000 & 0.369 \\
\hline PDK3 & 4.825 & $2.099-11.092$ & 0.000 & 0.202 \\
\hline PDSS1 & 22.868 & $6.255-83.603$ & 0.000 & 0.419 \\
\hline RAD51C & 16.936 & $3.992-71.847$ & 0.000 & 0.128 \\
\hline TOMM34 & 61.837 & $8.836-432.757$ & 0.000 & 0.028 \\
\hline TRMU & 38.376 & $7.287-202.108$ & 0.000 & 1.033 \\
\hline
\end{tabular}

Page $4 / 19$ 
The risk scores were calculated using the formula mentioned above, patients in every cohort were further divided into high and low-risk groups with the cut-off value of the median risk score. And the expression levels of every biomarker in different groups were analyzed (Fig. $2 \mathrm{~b}$ ).

Additionally, the levels of ACOT7, ADPRHL2, ATAD3A, BSG, FAM72A, PDSS1, RAD51C, and TOMM34 in the classifier were much higher in HCC than normal liver tissues (Fig. 2c). Besides, the correlation between these genes and clinical characteristics showed that these genes were positively correlated with the progression of HCC and negatively correlated with HCC prognosis (Fig. 2d).

\subsection{Correlation Between Classifiers and Clinicopathologic Characteristics}

As shown in Table 2, the clinical characteristics of gender, age, child-pugh grade, and pathologic M showed no significant differences between the high and low-risk groups in the training, validation, and total cohort. However, BMI, histologic grade, Pathologic T (pT), Pathologic N (pN), and tumor stage showed significant difference between the two groups. 
Table 2

Correlations between risk score of the mitochondrial-related genes-based classifier with clinicopathological characteristics in training cohort, validation cohort and total cohort

\begin{tabular}{|c|c|c|c|c|c|c|c|c|c|c|c|c|}
\hline \multirow[t]{2}{*}{ Parameters } & \multicolumn{4}{|c|}{ Training cohort } & \multicolumn{4}{|c|}{ Validation cohort } & \multicolumn{4}{|c|}{ Total cohort } \\
\hline & $\begin{array}{l}\text { High } \\
\text { risk }\end{array}$ & $\begin{array}{l}\text { Low } \\
\text { risk }\end{array}$ & $x^{2}$ & $P$ & $\begin{array}{l}\text { High } \\
\text { risk }\end{array}$ & $\begin{array}{l}\text { Low } \\
\text { risk }\end{array}$ & $x^{2}$ & $P$ & $\begin{array}{l}\text { High } \\
\text { risk }\end{array}$ & $\begin{array}{l}\text { Low } \\
\text { risk }\end{array}$ & $x^{2}$ & $P$ \\
\hline Age (y) & & & 0.908 & 0.341 & & & 0.088 & 0.766 & & & 0.958 & 0.439 \\
\hline$<60$ & 49 & 42 & & & 38 & 40 & & & 89 & 82 & & \\
\hline$>60$ & 45 & 51 & & & 55 & 53 & & & 98 & 106 & & \\
\hline Gender & & & 0.014 & 0.905 & & & 1.328 & 0.249 & & & 0.990 & 0.320 \\
\hline Male & 68 & 68 & & & 55 & 62 & & & 122 & 131 & & \\
\hline Female & 26 & 25 & & & 39 & 31 & & & 65 & 56 & & \\
\hline $\begin{array}{l}\text { Child-pugh } \\
\text { grade }\end{array}$ & & & 0.514 & 0.473 & & & 0.729 & 0.393 & & & & \\
\hline A & 33 & 56 & & & 62 & 68 & & & 92 & 127 & 0.010 & 0.921 \\
\hline$B$ and $C$ & 4 & 4 & & & 5 & 9 & & & 9 & 13 & & \\
\hline BMI & & & 0.625 & 0.429 & & & 6.798 & 0.009 & & & 5.822 & 0.016 \\
\hline$\varangle 28$ & 60 & 54 & & & 79 & 63 & & & 151 & 130 & & \\
\hline$\geq 28$ & 22 & 26 & & & 15 & 30 & & & 37 & 57 & & \\
\hline $\begin{array}{l}\text { Histologic } \\
\text { grade }\end{array}$ & & & 14.321 & 0.000 & & & 10.957 & 0.001 & & & 13.755 & 0.000 \\
\hline $1-2$ & 61 & 81 & & & 34 & 57 & & & 99 & 134 & & \\
\hline $3-4$ & 32 & 10 & & & 58 & 36 & & & 85 & 51 & & \\
\hline $\mathrm{pT}$ & & & 6.376 & 0.012 & & & 0.097 & 0.756 & & & 4.779 & 0.029 \\
\hline $1-2$ & 60 & 73 & & & 72 & 73 & & & 131 & 147 & & \\
\hline $3-4$ & 34 & 17 & & & 22 & 20 & & & 56 & 37 & & \\
\hline $\mathrm{pN}$ & & & 0.003 & 0.956 & & & 2.026 & 0.155 & & & 4.047 & 0.044 \\
\hline 0 & 52 & 48 & & & 76 & 78 & & & 125 & 129 & & \\
\hline 1 & 1 & 1 & & & 2 & 0 & & & 4 & 0 & & \\
\hline pM & & & - & - & & & 0.944 & 0.331 & & & 0.953 & 0.329 \\
\hline 0 & 57 & 56 & & & 77 & 78 & & & 133 & 135 & & \\
\hline 1 & 0 & 0 & & & 1 & 3 & & & 1 & 3 & & \\
\hline Tumor stage & & & 5.176 & 0.023 & & & 0.773 & 0.392 & & & 6.925 & 0.012 \\
\hline $1-2$ & 53 & 66 & & & 68 & 73 & & & 119 & 141 & & \\
\hline $3-4$ & 29 & 16 & & & 25 & 20 & & & 55 & 35 & & \\
\hline
\end{tabular}

\subsection{Prognostic Value of Classifiers for Assessing Overall Survival}

As shown in Fig. 3a-c, with the increase of risk score, the survival time of patients is decreased, and almost all the dead patients were enrolled in the high-risk group.

To further assess the prognostic value of the classifier, Kaplan-Meier test was conducted. As shown in Fig. 3d-f, patients in high-risk group had significantly unfavorable prognosis.

Besides, the results of univariate Cox regression analysis in training, validation, and total cohorts further validated the prognostic value of classifier (Table 3). Moreover, the multivariate analysis suggested that the classifier was the independent risk factors of survival for HCC 
patients (Table 3).

Table 3

Univariate and multivariate Cox regression analysis of clinicopathologic factors for overall survival in LIHC from TCGA database

\begin{tabular}{|c|c|c|c|c|c|c|c|c|c|c|c|c|}
\hline \multirow[t]{3}{*}{ Items } & \multicolumn{4}{|c|}{ Training cohort } & \multicolumn{4}{|c|}{ Validation cohort } & \multicolumn{4}{|c|}{ Total cohort } \\
\hline & \multicolumn{2}{|c|}{ Univariate cox } & \multicolumn{2}{|c|}{$\begin{array}{l}\text { Multivariate } \\
\text { cox }\end{array}$} & \multicolumn{2}{|c|}{ Univariate cox } & \multicolumn{2}{|c|}{$\begin{array}{l}\text { Multivariate } \\
\text { cox }\end{array}$} & \multicolumn{2}{|c|}{ Univariate cox } & \multicolumn{2}{|c|}{$\begin{array}{l}\text { Multivariate } \\
\text { cox }\end{array}$} \\
\hline & HR & $\mathbf{P}$ & HR & $\mathbf{P}$ & HR & $\mathbf{P}$ & HR & $\mathbf{P}$ & HR & $\mathbf{P}$ & HR & $\mathbf{P}$ \\
\hline Gender & 1.313 & 0.315 & & & 1.319 & 0.269 & & & 1.260 & 0.201 & & \\
\hline Age & 1.015 & 0.125 & & & 1.012 & 0.245 & & & 1.012 & 0.091 & & \\
\hline $\begin{array}{l}\text { Child } \\
\text { grade }\end{array}$ & 1.335 & 0.619 & & & 1.768 & 0.141 & & & 1.543 & 0.157 & & \\
\hline BMI & 0.963 & 0.145 & & & 1.027 & 0.123 & & & 1.000 & 0.998 & & \\
\hline $\begin{array}{l}\text { Histologic } \\
\text { grade }\end{array}$ & 1.041 & 0.834 & & & 1.276 & 0.158 & & & 1.104 & 0.410 & & \\
\hline pT & 2.091 & 0.000 & 2.809 & 0.127 & 1.358 & 0.020 & 1.227 & 0.662 & 1.683 & 0.000 & 1.655 & 0.213 \\
\hline $\mathrm{pN}$ & 4.473 & 0.144 & & & 1.274 & 0.811 & & & 2.029 & 0.324 & & \\
\hline pM & - & - & & & 4.190 & 0.017 & 2.777 & 0.151 & 4.077 & 0.017 & 2.131 & 0.254 \\
\hline $\begin{array}{l}\text { Tumor } \\
\text { stage }\end{array}$ & 2.158 & 0.000 & 0.612 & 0.495 & 1.363 & 0.015 & 1.019 & 0.968 & 1.638 & 0.000 & 0.955 & 0.913 \\
\hline RiskScore & 6.681 & 0.000 & 4.544 & 0.000 & 2.390 & 0.003 & 2.106 & 0.031 & 3.941 & 0.000 & 3.218 & 0.000 \\
\hline
\end{tabular}

In addition, in the time-dependent ROC curve analysis, the Area Under The Curve (AUC) for overall survival in the 1-, 3-, and 5- year was 0.838, 0.771 , and 0.834 in the training cohort, $0.716,0.627$, and 0.608 in the validation cohort, and $0.787,0.696$, and 0.705 in the total cohort (Fig. 4). Moreover, the prediction capability of the classifier seems superior to histologic grade and tumor grade, which may be two major risk factors for tumor prognosis as reported by previous studies.

The results above indicated that the Mito-RGs based classifier provided a useful prognostic tool with clinical value for appropriately categorizing patients with HCC.

\subsection{Pathway Enrichment Analysis}

We performed GSEA analysis to investigate biological pathways correlated with metabolism between high and low-risk groups. The results showed that low-risk HCC was significantly enriched in metabolic pathways of Arginine biosynthesis, Beta-alanine metabolism, Citrate cycle (TCA cycle), Fatty acid metabolism, Fatty acid degradation, Glycine, serine and threonine metabolism, Histidine metabolism, Primary bile acid biosynthesis, Pyruvate metabolism, Tyrosine metabolism, Tryptophan metabolism, and Valine, leucine and isoleucine degradation (Fig. 5a). Besides, GSVA analysis showed that pathways of Fatty acid degradation, Fatty acid metabolism, Beta-alanine metabolism, Glycine serine and threonine metabolism, Histidine metabolism, Tryptophan metabolism, Tyrosine metabolism, Valine leucine and isoleucine degradation, Linoleic acid metabolism, Retinol metabolism, Primary bile acid biosynthesis, and Drug metabolism cytochrome p450 were significantly downregulated in high-risk HCC (Fig. 5b).

Additionally, we conducted Kaplan-Meier analyses to further verify the value of these metabolic processes in the prognosis of HCC. As shown in Fig. 5c, high level of Fatty acid metabolism, Glycine serine and threonine metabolism, Histidine metabolism, Linoleic acid metabolism, Primary bile acid biosynthesis, Tyrosine metabolism, Tryptophan metabolism, and Valine leucine and isoleucine degradation correlated with favorable prognosis. We further investigated the gene expressions associated with these prognostic metabolic pathways. As shown in Additional files 2: Supplementary Fig. 2, most genes were downregulated in these pathways.

We also compared the activity of these pathways between HCC and normal liver tissues, as shown in Fig. $5 \mathrm{~d}$ and Additional files 2 : Supplementary Fig. 3, these pathways and most associated genes were downregulated in HCC compared with normal liver tissues.

The results above indicated that the downregulation of these metabolic processes about fatty acid, amino acid, and bile acid may play crucial roles in the initiation and progression of HCC.

\subsection{Metabolic subgrouping}


In order to further investigated the role of these metabolic processes in HCC, we conducted cluster analysis based on different metabolic genesets. As shown in Fig. 6a, HCC tissues were distinctly divided into 2 subgroups based on genes belonging to KEGG geneset "fatty acid metabolism". Besides, patients in cluster 2 share a favorable prognosis with a higher level of metabolic processes about fatty acid, amino acid, and bile acid (Fig. 6b and 6c).

Similarly, HCC tissues were distinctly divided into 2 subgroups based on genes belonging to KEGG genesets "Glycine, serine, and threonine metabolism", "Histidine metabolism”, "Tryptophan metabolism”, "Tyrosine metabolism”, and "Valine, leucine, and isoleucine degradation" (Fig. 6d). Besides, patients in cluster 2 share favorable prognosis with higher level of metabolic processes about fatty acid, amino acid, and bile acid (Fig. 6e d 6f).

Additionally, HCC tissues were distinctly divided into 3 subgroups based on genes belonging to KEGG genesets "Primary bile acid biosynthesis" (Fig. 6g). Besides, patients in cluster 2, and cluster 3 share a favorable prognosis and with higher level of metabolic processes about fatty acid, amino acid, and bile acid (Fig. 6h and 6i).

The results above further demonstrated that metabolic processes about fatty acid, amino acid, and bile acid play roles in the prognosis of HCC.

\section{Discuss}

HCC is one of the leading causes of cancer-related deaths with highly malignant, recurrent, metastatic, drug-resistant, and late diagnosis [12]. Thus, the identification of effective biomarkers for HCC-specific prognosis is urgently needed to improve the management for patients. Recently, global alterations in metabolic pathways were identified in HCC [13], providing some new diagnostic and therapeutic opportunities [14]. Taking into account the importance of the alteration of metabolic processes in HCC progression and the highly central position of the mitochondrion in human metabolism, it is essential to identify mitochondrial-related biomarkers for the prognosis of HCC patients, which may also help us to clarify underlying metabolism alterations and identify potential therapeutic drugs, so as to bring new insights into the improvement of the prognosis of HCC patients.

In the present study, a 10 Mito-RGs based prognostic classifier for HCC was constructed and validated to optimize the predictive capacity of prognosis for HCC patients for the first time. The classifier did well in predicting the prognosis for HCC patients in training, validation, and total cohorts, indicating the repeatability and practicability of the classifier for the prognostic prediction for HCC overall survival. Besides, the prediction efficacy of the classifier was superior to histologic grade and tumor stage (TNM stage), which may be two major risk factors for tumor prognosis as reported by previous studies $[15,16]$.

All these 10 Mito-RGs of the classifier, ACOT7, ADPRHL2, ATAD3A, BSG, FAM72A, PDK3, PDSS1, RAD51C, TOMM34, and TRMU, were riskassociated, and higher expressed in high-risk group. Besides, among them, ACOT7, ADPRHL2, ATAD3A, BSG, FAM72A, PDSS1, RAD51C, and TOMM34 were overexpressed in HCC compared with normal liver tissues, indicating potential roles of these genes in the initiation and development of HCC. ACOT7 is an isoform of the ACOT family, which responsible for cleaving fatty acyl-CoAs to free fatty acids that involved in responses to endoplasmic reticulum stress in lung cancer [17]. Besides, the high expression of ACOT7 indicates unfavorable outcome in acute myeloid leukemia patients [18]. ADPRHL2, also known as ADP-ribosylhydrolase 3 (ARH3), is the main hydrolase for catalyzing the hydrolysis of ADP-ribosylated serine. The deficiency of ADPRHL2 will enhance the tumor-suppression effect of ARH1 [19], indicating the potential carcinogenic role of ADPRHL2. ATPase family AAA domain-containing protein 3 (ATAD3A) is a mitochondrial membrane-bound ATPase, involving in various cellular processes [20]. Besides, the high expression of ATAD3A is correlated with the poor prognosis of HCC [21]. BSG, also termed as CD147, is a member of the immunoglobulin superfamily that is overexpressed and positively correlated with HCC malignant potential and poor prognosis [22]. FAM72A, also known as p17, is a novel neuronal protein that also exerts tumorigenic effects in multiple tissues [23] by modulating cell survival and promoting the cell cycle [24]. RAD51C is one of the paralogs of RAD51 and is essential for homologous recombination [25]. High expression of RAD51C predicts poor prognosis and is correlated with resistance to chemoradiotherapy in lung cancer [26]. TOMM34 is a kind of protein located in the outer membrane of mitochondria (TOMM), which overexpressed in breast cancer and served as a biomarker of the progression and poor prognosis of breast cancer [27]. However, the role of these genes in HCC mostly unclear. Considering the strong relevance to the prognosis of HCC, these genes highly worthy of further investigation in HCC.

Metabolic alterations are a well-founded hallmark of HCC [28]. The wide range of metabolic alterations is highly associated with the heterogeneity of HCC, providing challenges for the clinical management of HCC patients [6]. In the present study, we found that the metabolic pathways about fatty acid metabolism, amino acid metabolism, and bile acid biosynthesis were downregulated in high-risk HCC patients, indicating the potential role of these metabolic processes in the progression of HCC.

The dysregulation of de novo lipogenesis is a well-known characteristic of cancer metabolism, which ensures the energy supply for the proliferating tumor cells [29]. The liver has a central role in the processes of lipids synthesis, storage, and degradation. The imbalance of fatty acid metabolism induced by upregulation of de novo lipogenesis and downregulation of lipid degradation in the liver will lead to the 
accumulation of lipids, and can rapidly develop the nonalcoholic fatty liver disease (NAFLD), which is a risk factor for HCC [30]. Studies showed that the upregulation of the lipogenic metabolism genes, including fatty acids biosynthesis enzymes (ACAC, ACLY, FASN, and SCD1), enzymes for cholesterol biosynthesis (HMGCR, MVK, SREBP2, and SQS), as well as their upstream transcriptional factors (chREBP, LXR- $\beta$, and SREBP1) was observed in HCC when compared with adjacent normal liver tissues [31]. Besides, the higher levels of lipogenic enzymes were associated with poorer prognosis [31]. In the present study, we also found the metabolic process of fatty acid degradation was downregulated in high-risk group of HCC, and its high level correlated with a better prognosis of HCC patients. These data indicated that increased lipogenesis and downregulated lipid degradation will promote the development and progression of HCC, which may be potential therapeutic targets for HCC.

Amino acids (AAs) perform important metabolic functions, which can be divided into essential AAs, including histidine, isoleucine, lysine, leucine, methionine, phenylalanine, tryptophan, threonine, and valine, semi-essential AAs, including arginine, cysteine, glutamine, glycine, tyrosine, and proline, non-essential AAs, including alanine, aspartate, asparagine, glutamate, and serine [32]. They are not only components for protein synthesis but also intermediate metabolites for multiple biosynthetic pathways. Besides, increasing studies have shown that AAs also functioned as metabolites and metabolic regulators in supporting cancer cell growth [32].

The alterations of AAs metabolism were characterized in HCC compared to other liver diseases. Research has shown that the alteration of urine metabolites in HCC patients was mainly involved in arginine and proline metabolism, glutathione metabolism, phenylalanine metabolism, and tyrosine metabolism, which may be considered as potential biomarkers [33]. Metabolites of AAs such as tyrosine (OR $=4.11)$, glutamate $(O R=3.93)$, glutamine $(O R=0.15)$, kynurenine $(O R=3.32)$, lysine $(O R=0.35)$, and leucine $(O R=0.19)$, isoleucine $(O R=0.51)$, valine $(O R=0.56)$, $A A$ groups and metabolite ratios such as branched-chain $(O R=0.26)$, glucogenic $(O R=0.25)$ and aromatic $A A(O R=3.29)$, and the Fischer's ratio (branched-chain to aromatic $A A s)(O R=0.10)$, glutamate/glutamine $(O R=5.49)$ and kynurenine/tryptophan $(O R=2.29)$ ratios were found significantly associated with HCC risk [34]. In the present study, the pathways of Glycine, serine and threonine metabolism, Histidine metabolism, Tyrosine metabolism, Tryptophan metabolism, and Valine, leucine and isoleucine degradation were found downregulated in high risk of HCC, and low level of these metabolic processes was correlated with poor prognosis.

Serine and glycine are linked in the biosynthetic process that serine is formed prior the glycine. They together provide the essential precursors for glucose, lipids, proteins, and nucleic acids synthesis through one-carbon metabolism that are crucial to cancer cell growth and support tumor homeostasis [35]. The upregulation of serine/glycine metabolism promotes cell proliferation and correlates with poor prognosis in various tumors [36, 37]. Metformin has recently acted as a promising agent for cancer therapy. A recent study revealed that metformin exerts anti-tumor effects through the alteration of one-carbon metabolism [38].

Tyrosine metabolism has been found altered in esophageal squamous cell carcinoma (ESCC) patients and the metabolites involved in can be utilized as diagnostic biomarkers [39]. Besides, studies showed that 4-hydroxyphenylpyruvate dioxygenase (HPD) played important roles in tumorigenesis through promoting tyrosine metabolism [40]. Researches showed that activation of tyrosine metabolism in CD13 ${ }^{+}$cancer stem cells triggers the relapse of HCC [41].

Tryptophan metabolism is involved in the regulation of immune function, neuronal function, and intestinal homeostasis. Studies have shown that tryptophan metabolism played important roles in promoting tumor progression by contributing to tumor immune escape and increasing the malignancy of cancer cells [42]. Indoleamine 2,3 dioxygenase 1 (ID01) is the most important enzymes that initiate the catabolism of tryptophan to kynurenine, which is expressed in 58\% of human tumors [43], and its expression is correlated with poor prognosis in various cancers including melanoma, colorectal cancer, ovarian cancer, brain tumors, and acute myelogenous leukemia [44].

Leucine, isoleucine, and valine are essential AAs of branched-chain amino acids (BCAAs), and they have recently served as predictive factors for the risk of cancers [45]. BCAAs metabolism is altered in various tumors, including melanoma, breast cancer, and nasopharyngeal carcinoma [45]. BCAAs are thought to play important roles in cancer cells through activating of the mammalian target of rapamycin complex 1 (mTORC1), which promoting protein synthesis, cell growth, and proliferation [46]. Besides, they can also provide the essential precursors for nucleic acids synthesis and tricarboxylic acids cycle (TCA) that contribute to energy production for cancer cell growth [47]. Additionally, the role of branched-chain aminotransferase 1 (BCAT1), the key enzyme that mediates BCAAs catabolism, played essential roles in cancer progression. In breast cancer [48], lung cancer [49], ovarian cancer [50], HCC [51], and chronic myeloid leukemia [52], BCAT1 levels are increased compared to normal tissues and promote cell proliferation through activating $\mathrm{mTORC1}$, inhibiting autophagy, suppression isocitrate dehydrogenase 1(IDH1), IDH2, and aldo-keto reductase family 1 member C1 (AKR1C1). Besides, a recent meta-analysis revealed that BCAAs supplementation improved liver functional, reduced 3-year mortality for HCC patients [53].

Primary bile acids are synthesized from cholesterol in the liver by the classic pathway and the alternative pathway. The classic pathway accounts for about $90 \%$ of total bile acid production in the liver (produces cholic acid (CA) and chenodeoxycholic acid (CDCA)), mainly catalyzed by cholesterol 7a-hydroxylase (CYP7A1) [54]. While, the alternative pathway is catalyzed by CYP27A1 and CYP7B1, produces 
chenodeoxycholic acid (CDCA) [54]. Early in the 1970s, a study has shown that plasma bile acid concentrations are elevated in HCC patients compared to healthy individuals [55], indicating that bile acid homeostasis was disturbed during HCC. Studies have shown that the knockout of whole body Farnesoid X receptor (FXR), which is an endogenous ligand for bile acids and transcriptional downregulates the expression of the enzymes of bile acid synthesis such as CYP7A1, CYP27A1, and CYP8B1 [56], would develop liver tumors in mice [57]. Increasing evidence revealed that bile acids could directly disrupt the plasma membrane and activate the MAPK-NF-KB pathway, resulting in the increase of TNF-a, IL-1 $\beta$ and IL-6. These cytokines could activate the JAK-STAT3 pathway, and PI3K-MDM2 pathway, which increases survival of DNA damaged cells and leading to the development of HCC. Besides, membrane injury by bile acids can also lead to the increase of reactive oxygen species (ROS) in the hepatocytes by activating cytosolic phospholipase A2 (PLA2), which can directly activate NF-KB and can also induce direct DNA damage in cells which might lead to HCC [58]. In the present study, we found that a high level of primary bile acid synthesis was associated with a better prognosis of HCC, which seems inconsistent with previous studies. The result may be due to the inhibitory role in proliferation and regeneration [59], and the induction of cell death by membrane disruptions [60] by the high concentration of bile acids, which may repress the progression of HCC. According to this, multiple synthetic bile acid derivatives were designed and found useful for cancer therapy [61].

Inevitably, the present study has some limitations. Firstly, it was a retrospective study based on the publicly online database. Secondly, the predictive role of our classifier was not confirmed by the cohort GSE76427 from GEO database (Gene Expression Omnibus) (Additional files 2: Supplementary Fig. 4). We further found that there existed a significant censoring proportion in the survival data of the GEO cohort. In which $14.8 \%$ patients were censored within 1 month, $35.7 \%$ within 1 year, and $47.8 \%$ within 2 years. Thus, we believed that our classifier could be used as a reliable prognostic predictor for HCC survival, since it was come from a large, high-quality database. Therefore, large-scale, multicenter studies are needed to verify our results before the Mito-RGs based classifier can be applied in the clinic.

\title{
5. Conclusion
}

In conclusion, we first identified and validated a classifier containing 10 Mito-RGs with independent prognostic significance for patients with HCC. Besides, we identified several metabolic pathways that correlated with the prognosis of HCC based on the classifier, indicating the potential anti-tumorous capacity of these metabolic pathway and relative metabolites. Moreover, our research may provide a novel method for HCC metabolic therapy based on the modulation of mitochondrial function.

\author{
Abbreviations \\ $\mathrm{HCC}$ \\ Hepatocellular carcinoma \\ TCGA \\ The Cancer Genome Atlas \\ Mito-RGs \\ Mitochondrial-related genes \\ WGCNA \\ Weighted gene coexpression network analysis \\ LASSO \\ The least absolute shrinkage and selection operation \\ GSVA \\ Gene set variation analysis \\ GSEA \\ Gene set enrichment analysis \\ ROC \\ Receiver operating characteristic \\ KEGG \\ Kyoto Encyclopedia of Genes and Genomes
}

\section{Declarations}

\section{AVAILABILITY OF DATA AND MATERIALS}

The data that support the findings of this study are available from the corresponding author upon reasonable request.

\section{COMPETING INTERESTS}

Page 10/19 
The authors declare that there are no conflicts of interest concerning this article.

ETHICS APPROVAL AND CONSENT TO PARTICIPATE

Not applicable

CONSENT FOR PUBLICATION

Not applicable

\section{FUNDING}

This work was supported by the National Natural Science Foundation of China (NO. 81570568, 81602419, and 81571075) and the National Key Research and Development Project (No. 2018YFC2001802).

\section{AUTHORS' CONTRIBUTIONS}

T.Z and Y.N: design, analysis, and interpretation of data, drafting of the manuscript; K.C: acquisition of data and statistical analysis; X.C, L.H, and J.W: critical revision of the manuscript for important intellectual content, obtaining funding, supervision. All authors read and approved the final manuscript.

\section{ACKNOWLEDGEMENTS}

The authors declare that there is no acknowledgement.

\section{References}

1. Siegel RL, Miller KD, Jemal A. Cancer statistics, 2019. CA Cancer J Clin. 2019;69(1):7-34.

2. Hamalainen RH. Mitochondria and mtDNA integrity in stem cell function and differentiation. Curr Opin Genet Dev. 2016;38:83-9.

3. Kim HK, Noh YH, Nilius B, Ko KS, Rhee BD, Kim N, Han J. Current and upcoming mitochondrial targets for cancer therapy. Semin Cancer Biol. 2017;47:154-67.

4. Valcarcel-Jimenez L, Gaude E, Torrano V, Frezza C, Carracedo A. Mitochondrial Metabolism: Yin and Yang for Tumor Progression. Trends Endocrinol Metab. 2017;28(10):748-57.

5. Weinberg SE, Chandel NS. Targeting mitochondria metabolism for cancer therapy. Nat Chem Biol. 2015;11(1):9-15.

6. Satriano L, Lewinska M, Rodrigues PM, Banales JM, Andersen JB. Metabolic rearrangements in primary liver cancers: cause and consequences. Nat Rev Gastroenterol Hepatol. 2019;16(12):748-66.

7. Langfelder P, Horvath S. WGCNA: an R package for weighted correlation network analysis. BMC Bioinformatics. 2008;9:559.

8. Zhang T, Guo J, Gu J, Chen K, Wang Z, Li H, Wang G, Wang J. KIAA0101 is a novel transcriptional target of FoxM1 and is involved in the regulation of hepatocellular carcinoma microvascular invasion by regulating epithelial-mesenchymal transition. $\mathrm{J}$ Cancer. 2019;10(15):3501-16.

9. Yu G, Wang LG, Han Y, He QY. clusterProfiler: an R package for comparing biological themes among gene clusters. OMICS. 2012;16(5):284-7.

10. Hanzelmann S, Castelo R, Guinney J. GSVA: gene set variation analysis for microarray and RNA-seq data. BMC Bioinformatics. $2013 ; 14: 7$.

11. Wilkerson MD, Hayes DN. ConsensusClusterPlus: a class discovery tool with confidence assessments and item tracking. Bioinformatics. 2010;26(12):1572-3.

12. Forner A, Reig M, Bruix J. Hepatocellular carcinoma. Lancet. 2018;391(10127):1301-14.

13. Nwosu ZC, Megger DA, Hammad S, Sitek B, Roessler S, Ebert MP, Meyer C, Dooley S. Identification of the Consistently Altered Metabolic Targets in Human Hepatocellular Carcinoma. Cell Mol Gastroenterol Hepatol. 2017;4(2):303-23 e301.

14. De Matteis S, Ragusa A, Marisi G, De Domenico S, Casadei Gardini A, Bonafe M, Giudetti AM. Aberrant Metabolism in Hepatocellular Carcinoma Provides Diagnostic and Therapeutic Opportunities. Oxid Med Cell Longev. 2018;2018:7512159.

15. Li R, Wang Y, Zhang X, Feng M, Ma J, Li J, Yang X, Fang F, Xia Q, Zhang Z, et al. Exosome-mediated secretion of LOXL4 promotes hepatocellular carcinoma cell invasion and metastasis. Mol Cancer. 2019;18(1):18. 
16. Ruan J, Zheng H, Rong X, Rong X, Zhang J, Fang W, Zhao P, Luo R. Over-expression of cathepsin B in hepatocellular carcinomas predicts poor prognosis of HCC patients. Mol Cancer. 2016;15:17.

17. Liu KT, Yeh IJ, Chou SK, Yen MC, Kuo PL. Regulatory mechanism of fatty acidCoA metabolic enzymes under endoplasmic reticulum stress in lung cancer. Oncol Rep. 2018;40(5):2674-82.

18. Zhang X, Liu B, Zhang J, Yang X, Zhang G, Yang S, Wang J, Shi J, Hu K, Wang J, et al. Expression level of ACOT7 influences the prognosis in acute myeloid leukemia patients. Cancer Biomark A. 2019;26(4):441-9.

19. Bu X, Kato J, Moss J. Emerging roles of ADP-ribosyl-acceptor hydrolases (ARHs) in tumorigenesis and cell death pathways. Biochem Pharmacol. 2019;167:44-9.

20. Frickey T, Lupas AN. Phylogenetic analysis of AAA proteins. J Struct Biol. 2004;146(1-2):2-10.

21. Liu X, Li G, Ai L, Ye Q, Yu T, Yang B. Prognostic value of ATAD3 gene cluster expression in hepatocellular carcinoma. Oncology letters. 2019;18(2):1304-10.

22. Tang X, Guo N, Xu L, Gou X, Mi M. CD147/EMMPRIN: an effective therapeutic target for hepatocellular carcinoma. J Drug Target. 2013;21(3):224-31.

23. Pramanik S, Kutzner A, Heese K. Lead discovery and in silico 3D structure modeling of tumorigenic FAM72A (p17). Tumour biology: the journal of the International Society for Oncodevelopmental Biology Medicine. 2015;36(1):239-49.

24. Heese K. The protein p17 signaling pathways in cancer. Tumour biology: the journal of the International Society for Oncodevelopmental Biology Medicine. 2013;34(6):4081-7.

25. Li N, Mclnerny S, Zethoven M, Cheasley D, Lim BWX, Rowley SM, Devereux L, Grewal N, Ahmadloo S, Byrne D, et al. Combined Tumor Sequencing and Case-Control Analyses of RAD51C in Breast Cancer. J Natl Cancer Inst. 2019;111(12):1332-8.

26. Chen X, Qian D, Cheng J, Guan Y, Zhang B, Ding X, Zeng J, Chen X, Er P, Zhang F, et al. High expression of Rad51c predicts poor prognostic outcome and induces cell resistance to cisplatin and radiation in non-small cell lung cancer. Tumour biology: the journal of the International Society for Oncodevelopmental Biology Medicine. 2016;37(10):13489-98.

27. Aleskandarany MA, Negm OH, Rakha EA, Ahmed MA, Nolan CC, Ball GR, Caldas C, Green AR, Tighe PJ, Ellis IO. TOMM34 expression in early invasive breast cancer: a biomarker associated with poor outcome. Breast Cancer Res Treat. 2012;136(2):419-27.

28. Hanahan D, Weinberg RA. Hallmarks of cancer: the next generation. Cell. 2011;144(5):646-74.

29. Zaidi N, Lupien L, Kuemmerle NB, Kinlaw WB, Swinnen JV, Smans K. Lipogenesis and lipolysis: the pathways exploited by the cancer cells to acquire fatty acids. Prog Lipid Res. 2013;52(4):585-9.

30. Lee J, Kim Y, Friso S, Choi SW. Epigenetics in non-alcoholic fatty liver disease. Mol Aspects Med. 2017;54:78-88.

31. Calvisi DF, Wang C, Ho C, Ladu S, Lee SA, Mattu S, Destefanis G, Delogu S, Zimmermann A, Ericsson J, et al. Increased lipogenesis, induced by AKT-mTORC1-RPS6 signaling, promotes development of human hepatocellular carcinoma. Gastroenterology. 2011;140(3):1071-83.

32. Fernandes HS, Silva Teixeira CS, Fernandes PA, Ramos MJ, Cerqueira NM. Amino acid deprivation using enzymes as a targeted therapy for cancer and viral infections. Expert Opin Ther Pat. 2017;27(3):283-97.

33. Zhang AH, Wang P, Sun H, Yan GL, Han Y, Wang XJ. High-throughput ultra-performance liquid chromatography-mass spectrometry characterization of metabolites guided by a bioinformatics program. Mol Biosyst. 2013;9(9):2259-65.

34. Stepien M, Duarte-Salles T, Fedirko V, Floegel A, Barupal DK, Rinaldi S, Achaintre D, Assi N, Tjonneland A, Overvad K, et al. Alteration of amino acid and biogenic amine metabolism in hepatobiliary cancers: Findings from a prospective cohort study. International journal of cancer. 2016;138(2):348-60.

35. Locasale JW. Serine, glycine and one-carbon units: cancer metabolism in full circle. Nature reviews Cancer. 2013;13(8):572-83.

36. Amelio I, Cutruzzola F, Antonov A, Agostini M, Melino G. Serine and glycine metabolism in cancer. Trends Biochem Sci. 2014;39(4):191-8.

37. Sun WY, Kim HM, Jung WH, Koo JS. Expression of serine/glycine metabolism-related proteins is different according to the thyroid cancer subtype. J Transl Med. 2016;14(1):168.

38. Corominas-Faja B, Quirantes-Pine R, Oliveras-Ferraros C, Vazquez-Martin A, Cufi S, Martin-Castillo B, Micol V, Joven J, Segura-Carretero A, Menendez JA. Metabolomic fingerprint reveals that metformin impairs one-carbon metabolism in a manner similar to the antifolate class of chemotherapy drugs. Aging. 2012;4(7):480-98.

39. Cheng J, Zheng G, Jin H, Gao X. Towards Tyrosine Metabolism in Esophageal Squamous Cell Carcinoma. Comb Chem High Throughput Screen. 2017;20(2):133-9.

40. Shan C, Lu Z, Li Z, Sheng H, Fan J, Qi Q, Liu S, Zhang S. 4-hydroxyphenylpyruvate dioxygenase promotes lung cancer growth via pentose phosphate pathway (PPP) flux mediated by LKB1-AMPK/HDAC10/G6PD axis. Cell Death Dis. 2019;10(7):525. 
41. Sun L, Zhang L, Chen J, Li C, Sun H, Wang J, Xiao H. Activation of Tyrosine Metabolism in CD13 + Cancer Stem Cells Drives Relapse in Hepatocellular Carcinoma. Cancer Res Treat 2019.

42. Platten M, Nollen EAA, Rohrig UF, Fallarino F, Opitz CA. Tryptophan metabolism as a common therapeutic target in cancer, neurodegeneration and beyond. Nature reviews Drug discovery. 2019;18(5):379-401.

43. Theate I, van Baren N, Pilotte L, Moulin P, Larrieu P, Renauld JC, Herve C, Gutierrez-Roelens I, Marbaix E, Sempoux C, et al: Extensive profiling of the expression of the indoleamine 2,3-dioxygenase 1 protein in normal and tumoral human tissues. Cancer immunology research 2015, 3(2):161-172.

44. Munn DH, Mellor AL. IDO in the Tumor Microenvironment: Inflammation, Counter-Regulation, and Tolerance. Trends Immunol. 2016;37(3):193-207.

45. Sivanand S, Vander Heiden MG. Emerging Roles for Branched-Chain Amino Acid Metabolism in Cancer. Cancer cell. 2020;37(2):147-56.

46. Ananieva EA, Powell JD, Hutson SM: Leucine Metabolism in T Cell Activation: mTOR Signaling and Beyond. Adv Nutr 2016, 7(4):798 s-805 $\mathrm{s}$.

47. Dey P, Baddour J, Muller F, Wu CC, Wang H, Liao WT, Lan Z, Chen A, Gutschner T, Kang Y, et al. Genomic deletion of malic enzyme 2 confers collateral lethality in pancreatic cancer. Nature. 2017;542(7639):119-23.

48. Zhang L, Han J. Branched-chain amino acid transaminase 1 (BCAT1) promotes the growth of breast cancer cells through improving mTOR-mediated mitochondrial biogenesis and function. Biochem Biophys Res Commun. 2017;486(2):224-31.

49. Mayers JR, Torrence ME, Danai LV, Papagiannakopoulos T, Davidson SM, Bauer MR, Lau AN, Ji BW, Dixit PD, Hosios AM, et al. Tissue of origin dictates branched-chain amino acid metabolism in mutant Kras-driven cancers. Science. 2016;353(6304):1161-5.

50. Wang ZQ, Faddaoui A, Bachvarova M, Plante M, Gregoire J, Renaud MC, Sebastianelli A, Guillemette C, Gobeil S, Macdonald E, et al. BCAT1 expression associates with ovarian cancer progression: possible implications in altered disease metabolism. Oncotarget. 2015;6(31):31522-43.

51. Zheng YH, Hu WJ, Chen BC, Grahn TH, Zhao YR, Bao HL, Zhu YF, Zhang QY. BCAT1, a key prognostic predictor of hepatocellular carcinoma, promotes cell proliferation and induces chemoresistance to cisplatin. Liver international: official journal of the International Association for the Study of the Liver. 2016;36(12):1836-47.

52. Hattori A, Tsunoda M, Konuma T, Kobayashi M, Nagy T, Glushka J, Tayyari F, McSkimming D, Kannan N, Tojo A, et al. Cancer progression by reprogrammed BCAA metabolism in myeloid leukaemia. Nature. 2017;545(7655):500-4.

53. Chen L, Chen Y, Wang X, Li H, Zhang H, Gong J, Shen S, Yin W, Hu H: Efficacy and safety of oral branched-chain amino acid supplementation in patients undergoing interventions for hepatocellular carcinoma: a meta-analysis. Nutr J 2015, 14:67.

54. Chiang JY. Bile acids: regulation of synthesis. J Lipid Res. 2009;50(10):1955-66.

55. Hirayama C, Irisa T. Serum cholesterol and bile acid in primary hepatoma. Clin Chim Acta. 1976;71(1):21-5.

56. Li T, Apte U. Bile Acid Metabolism and Signaling in Cholestasis, Inflammation, and Cancer. Adv Pharmacol. 2015;74:263-302.

57. Degirolamo C, Modica S, Vacca M, Di Tullio G, Morgano A, D'Orazio A, Kannisto K, Parini P, Moschetta A. Prevention of spontaneous hepatocarcinogenesis in farnesoid $X$ receptor-null mice by intestinal-specific farnesoid $X$ receptor reactivation. Hepatology. 2015;61(1):161-70.

58. Jia W, Xie G, Jia W. Bile acid-microbiota crosstalk in gastrointestinal inflammation and carcinogenesis. Nat Rev Gastroenterol Hepatol. 2018;15(2):111-28.

59. Zhang L, Huang X, Meng Z, Dong B, Shiah S, Moore DD, Huang W. Significance and mechanism of CYP7a1 gene regulation during the acute phase of liver regeneration. Molecular endocrinology (Baltimore Md). 2009;23(2):137-45.

60. Powell AA, LaRue JM, Batta AK, Martinez JD. Bile acid hydrophobicity is correlated with induction of apoptosis and/or growth arrest in HCT116 cells. Biochem J. 2001;356(Pt 2):481-6.

61. Kundu S, Kumar S, Bajaj A. Cross-talk between bile acids and gastrointestinal tract for progression and development of cancer and its therapeutic implications. IUBMB Life. 2015;67(7):514-23.

\section{Figures}


a

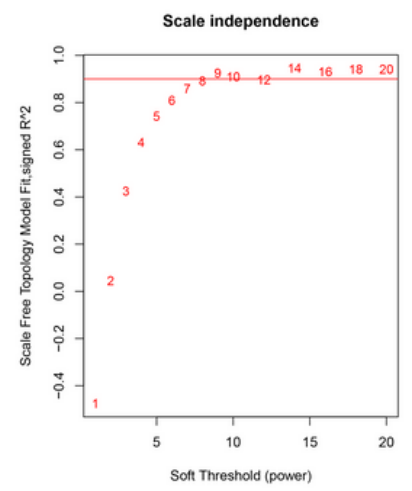

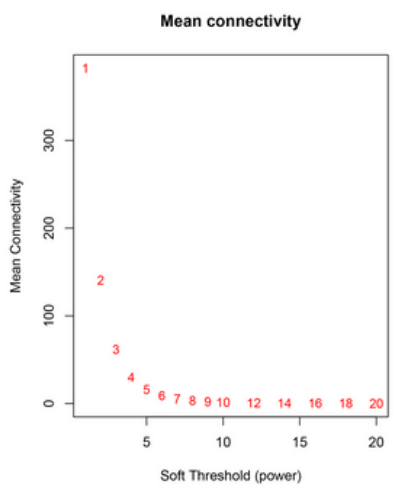

b

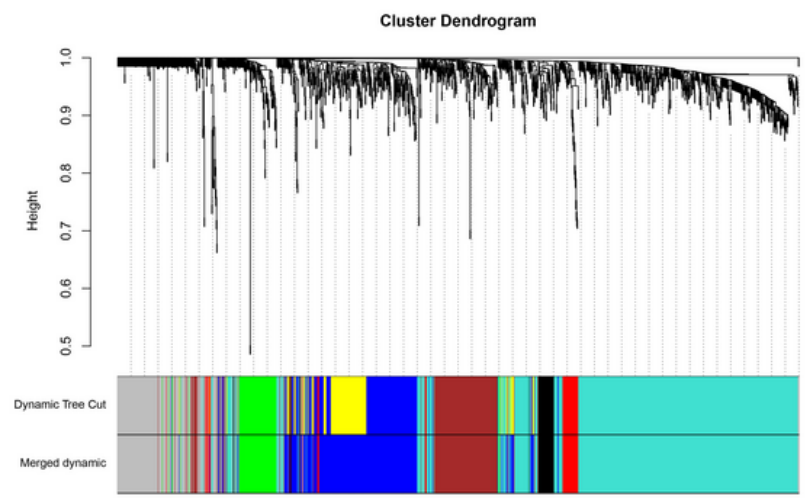

C

Module-trait relationships

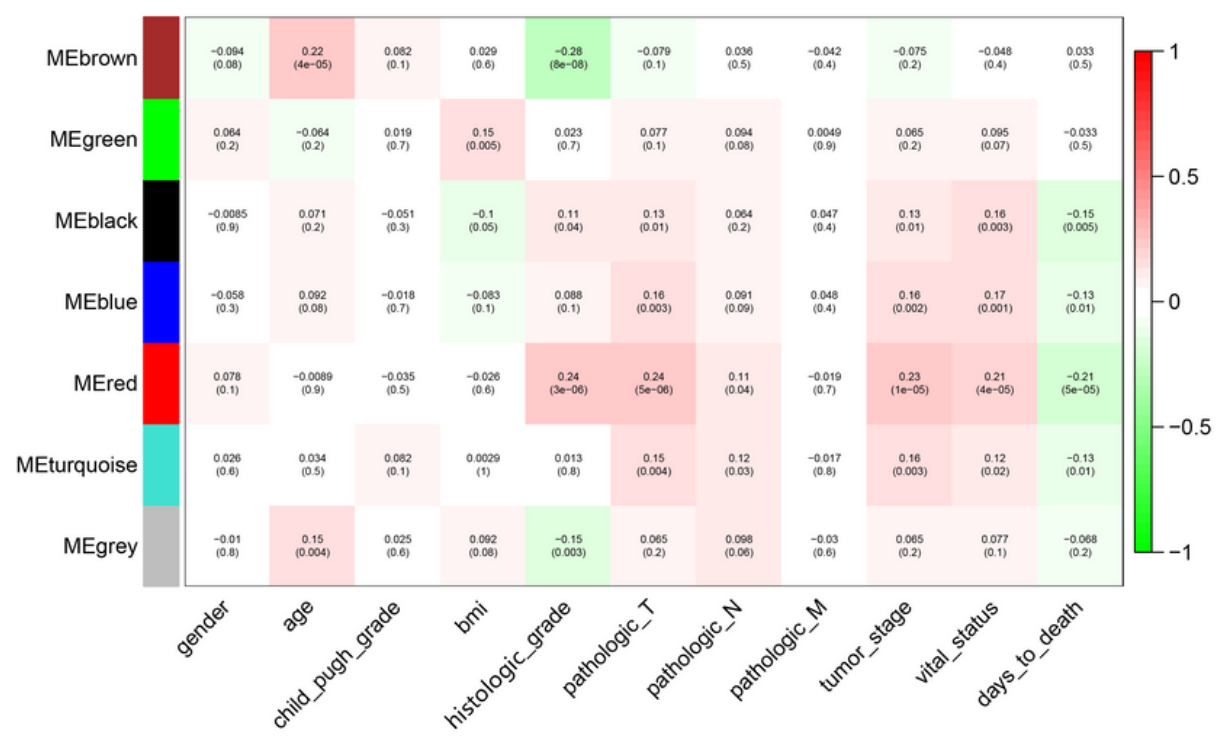

\section{Figure 1}

WGCNA network and module detection. (a) Selection of the soft-thresholding powers. Power 8 was chosen because the fit index curve flattened out upon reaching a high value (>0.9). (b) Cluster dendrogram and module assignment for modules from WGCNA. The colored horizontal bar represents the modules. (c) Correlation matrix for Eigengene values and clinical features. Each cell includes the corresponding correlations and the p-values. 
a
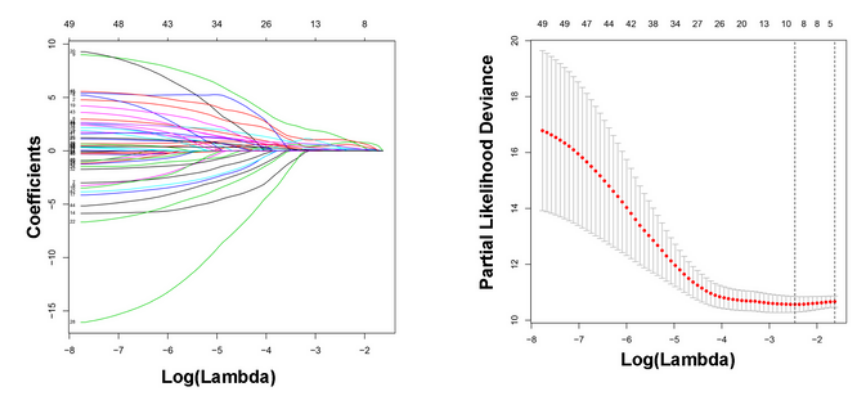

b
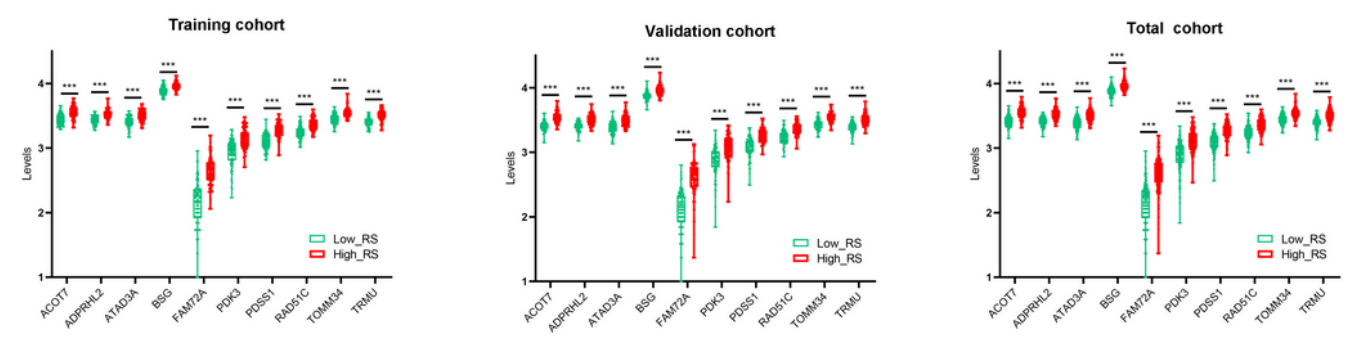

C
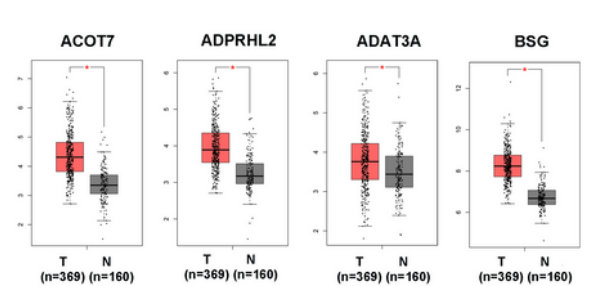

FAM72A

d
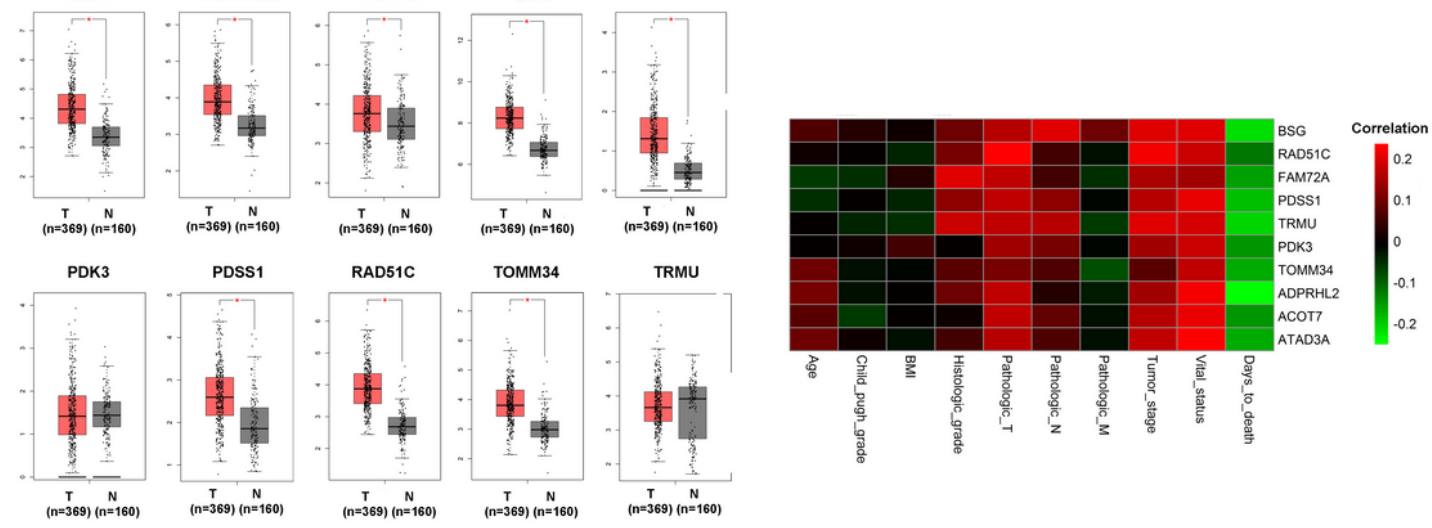

Figure 2

Construction of Mito-RGs based prognostic classifier. The results of the LASSO Cox regression suggested that all 10 genes were essential for the classifier (a). The expression levels of all 10 genes of the classifier in high and low-risk group from the training, validation, and total cohorts (b). The expression of the 10 genes in the classifier between HCC (T) and normal liver tissues (N) in GEPIA (http://gepia.cancer-pku.cn/) based on the TCGA and GTEx databases. ${ }^{*}<0.05$ (c). The correlation between the 10 genes in the classifier and the clinical features of HCC (d).

Abbreviations: RS: risk score. 
a
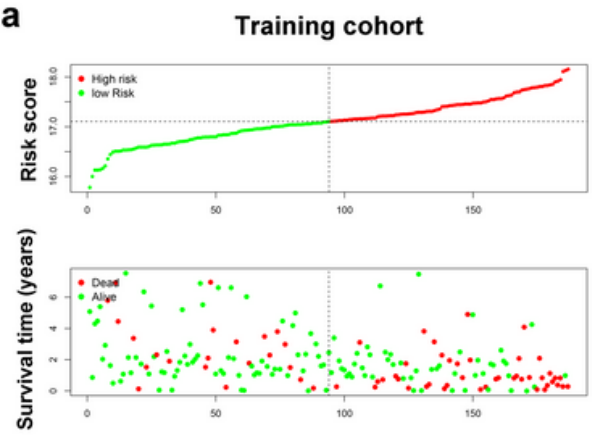

d
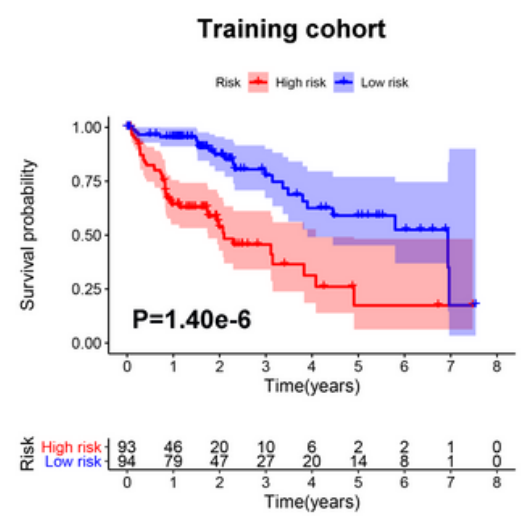

b
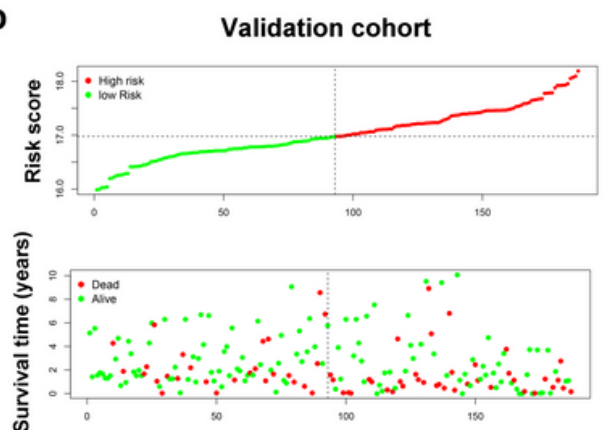

e

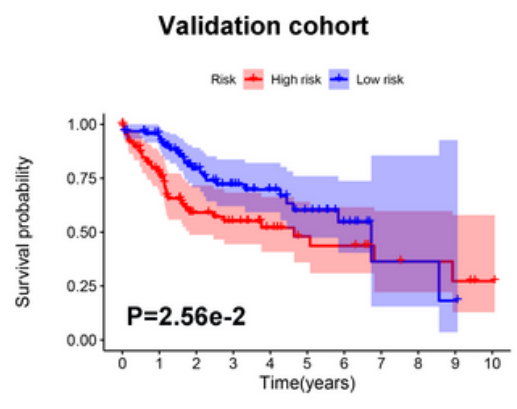

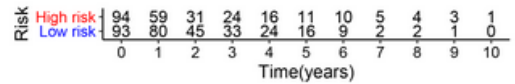

c
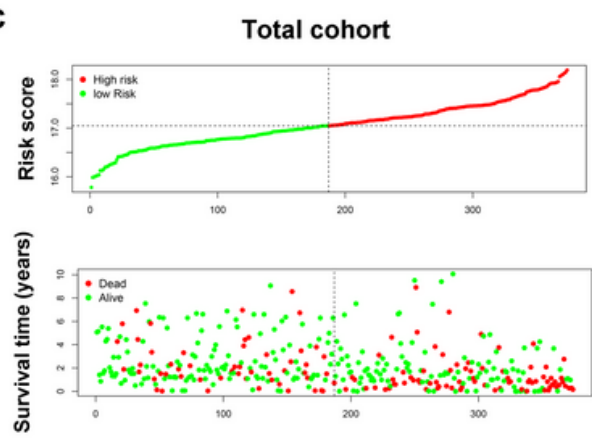

f

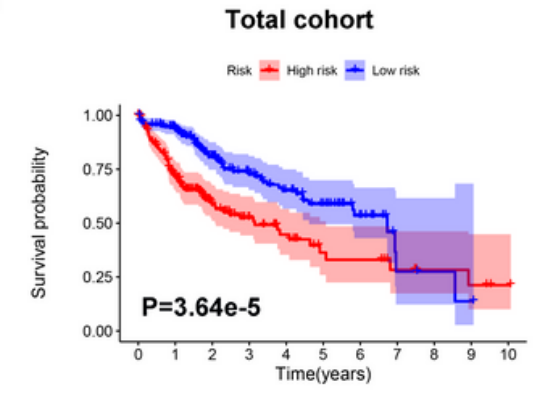

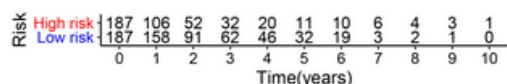

\section{Figure 3}

The prognostic value of Mito-RGs based classifier. The distribution of patients' risk score, survival state of the patients in high and low-risk group from training (a), validation (b), and total (c) cohorts. Kaplan-Meier survival analysis of overall survival between high and low-risk patients from training (d), validation (e), and total (f) cohorts. 
1 year OS
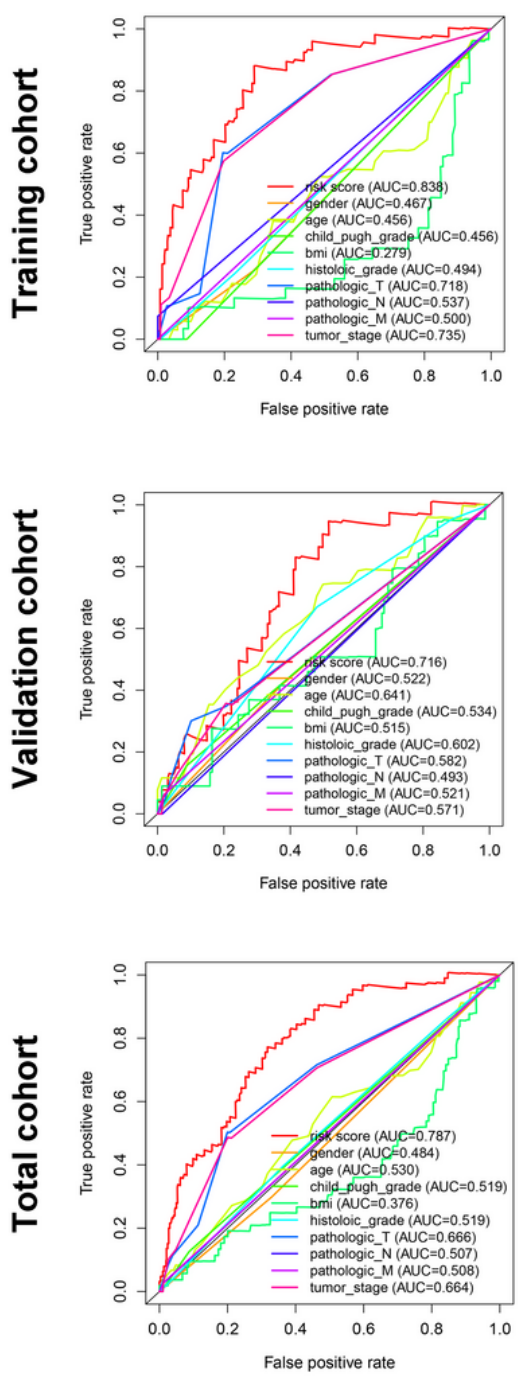

3 - year OS
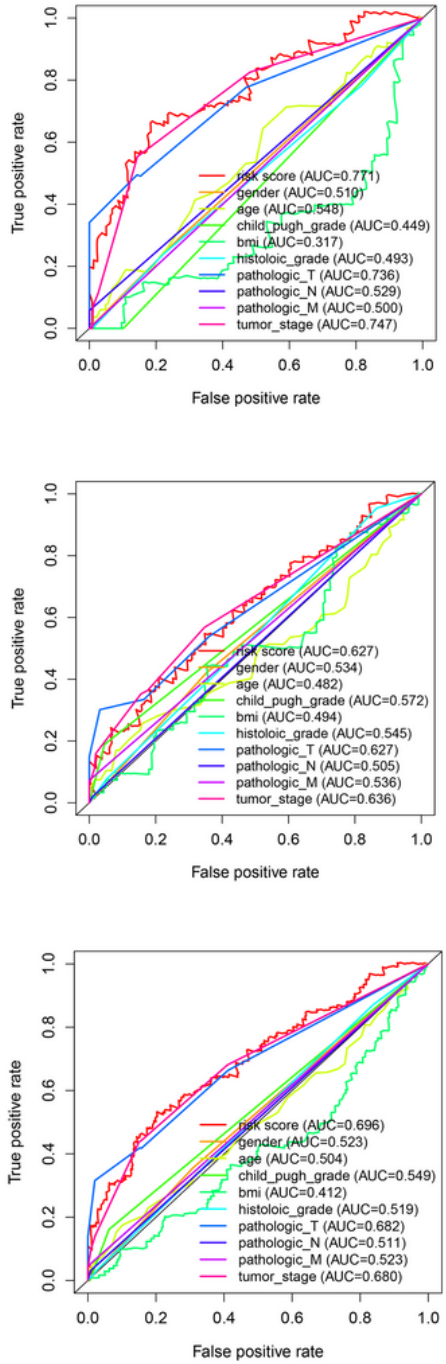

5 - year OS
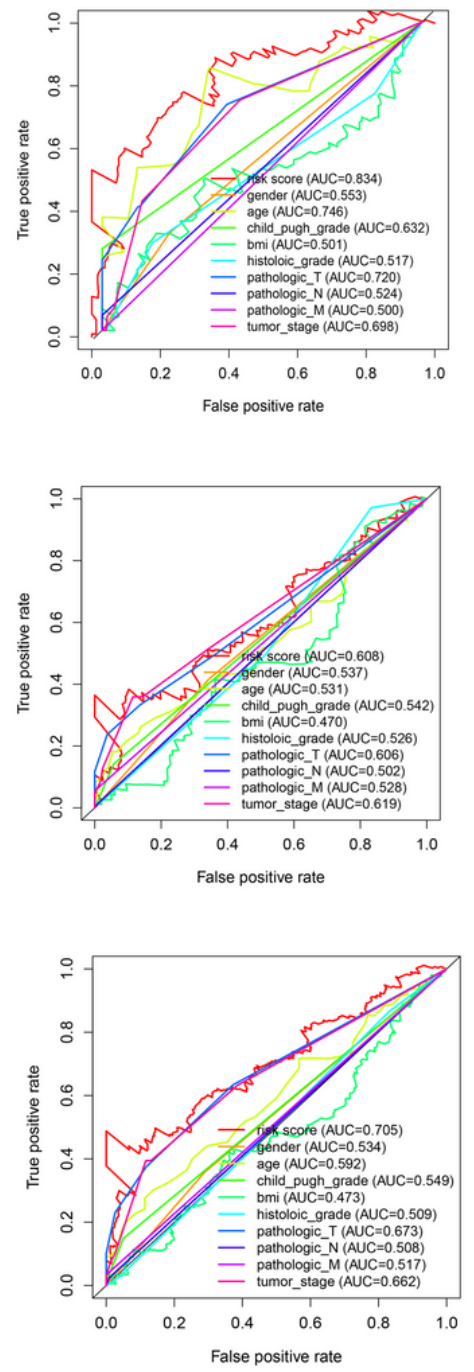

Figure 4

The time-dependent ROC for 1-, 3-, and 5-year overall survival predictions for the classifier in comparison with clinical features in the training, validation, and total cohorts of HCC. 
a

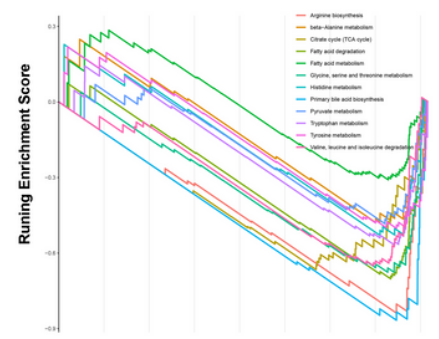

C FATTY ACID METABOLISM

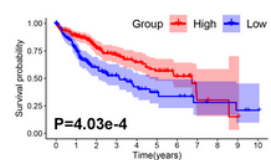

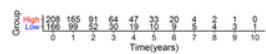

PRIMARY BILE ACID BIOSYNTHESIS

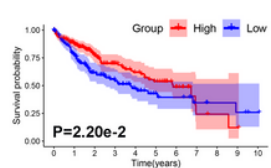

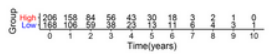

b

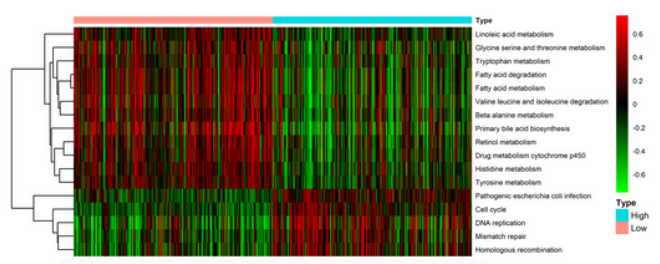

HISTIDINE METABOLISM

LINOLEIC ACID METABOLISM METABOLISM

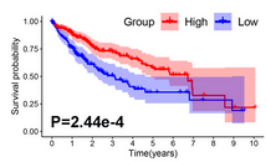

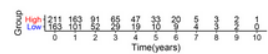

TRYPTOPHAN METABOLISM
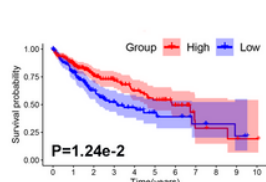

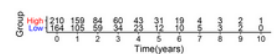

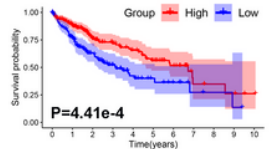

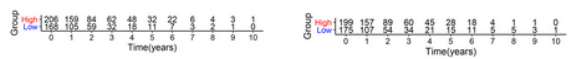

TYROSINE METABOLISM

VALINE LEUCINE AND ISOLEUCINE DEGRADATION

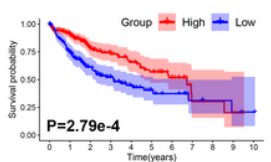

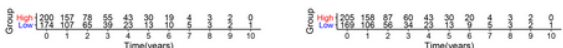

d
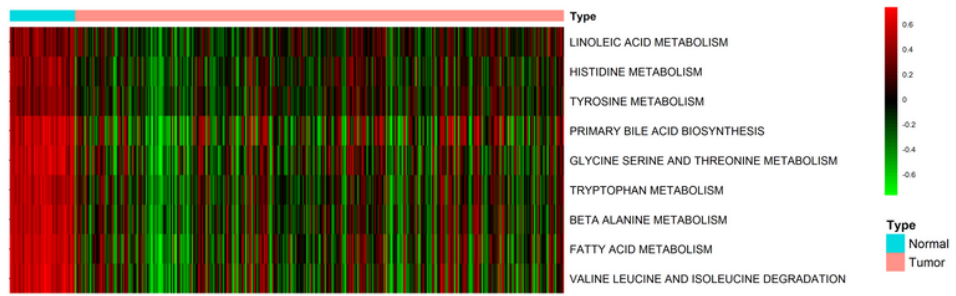

\section{Figure 5}

The alteration of metabolic processes between high and low-risk LIHC. (a) The metabolic pathways were significantly enriched in low-risk HCC by GSEA analysis. (b) The metabolic pathways were significantly downregulated in high-risk HCC by GSVA analysis. (c) Kaplan-Meier survival analysis of prognosis value of altered metabolic pathways in HCC. GSVA score $=0$ was set as the threshold value. (d) The prognostic metabolic pathways were also significantly downregulated in HCC compared with normal liver tissues by GSVA analysis. 
a

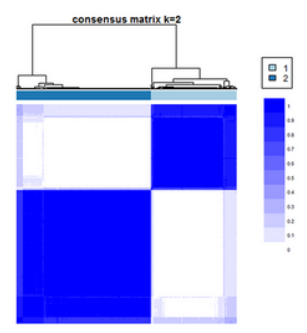

d

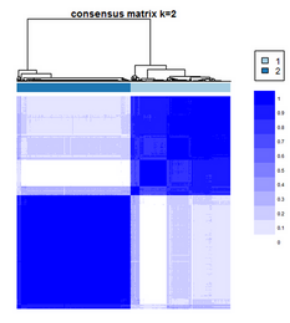

g

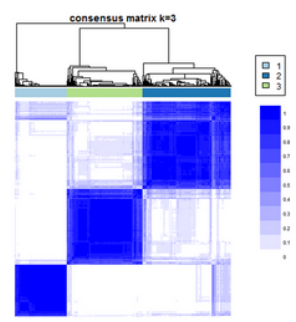

b

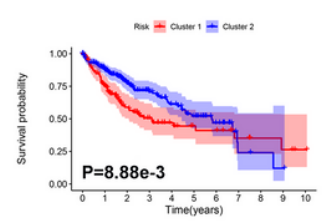

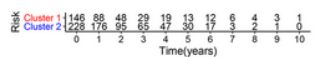

C

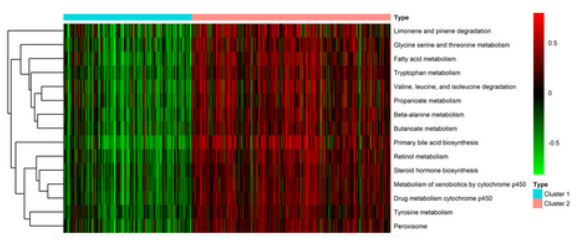

e

f

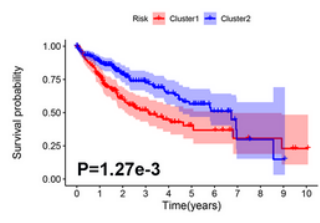

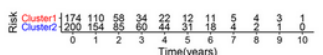

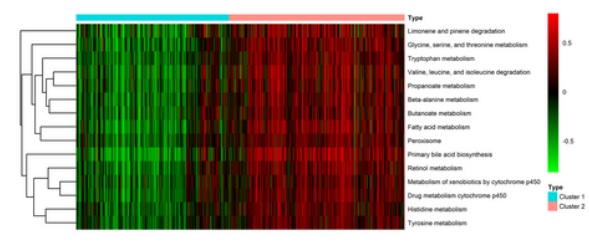

h

i

Cluster1 vs Cluster2
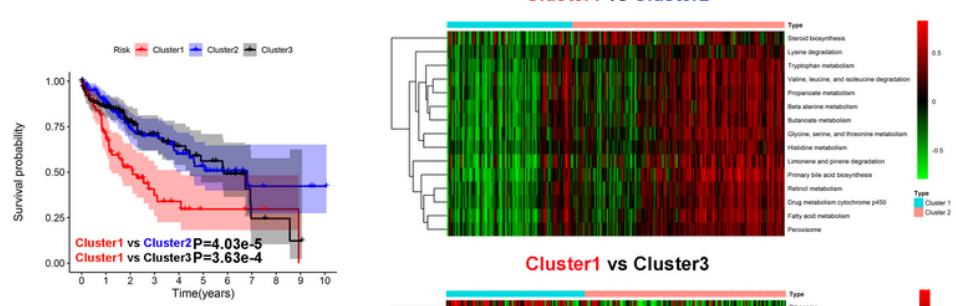

Cluster1 vs Cluster3

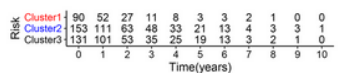

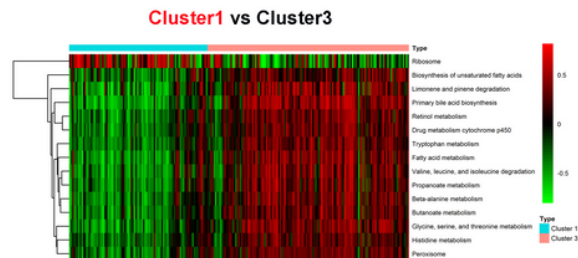

\section{Figure 6}

Metabolic subgroup of HCC based on the expression of metabolic genes. (a) Heatmap depicting consensus clustering solution ( $k=2$ ) for fatty metabolism genes in HCC. (b) Kaplan-Meier survival analysis of overall survival between clusters. (c) Top 15 altered KEGG pathways between clusters by GSVA analysis. (d) Heatmap depicting consensus clustering solution ( $k=2)$ for genes of 6 amino acid metabolism pathways in HCC. (e) Kaplan-Meier survival analysis of overall survival between clusters. (f) Top 15 altered KEGG pathways between clusters by GSVA analysis. (g) Heatmap depicting consensus clustering solution ( $k=3$ ) for genes of primary bile biosynthesis genes in HCC. ( $h$ ) Kaplan-Meier survival analysis of overall survival between clusters. (i) Top 15 altered KEGG pathways between clusters by GSVA analysis.

\section{Supplementary Files}

This is a list of supplementary files associated with this preprint. Click to download.

- Additionalfiles2.xlsx

- Additionalfiles1.docx 